\title{
Head-group Dependent Domain Morphologies in Langmuir Monolayers
}

\author{
M. M. Hossain*a and T. Kato ${ }^{b}$ \\ ${ }^{a}$ Department of Chemistry, University of Dhaka, Dhaka 1000, Bangladesh and \\ ${ }^{b}$ Venture Business Lab., Utsunomiya University, Utsunomiya 321-8585, Japan
}

\begin{abstract}
The effect of head groups on the thermodynamic properties and morphological features of the domains formed in the Langmuir monolayers at $10{ }^{\circ} \mathrm{C}$ has been investigated by a film balance and a Brewster angle microscopy (BAM). 2-hydroxyethyl myristate (2-HEM) and $n$-tetradecanoyl N-ethanolamide (NHEA-14), having the same chain length but different head groups have been taken as amphiphiles. Both the amphiphiles represent a first-order LE-LC phase transition at $10{ }^{\circ} \mathrm{C}$ at the air-water interface. The critical surface pressure necessary for this phase transition is $6.0 \mathrm{mN} / \mathrm{m}$ for 2-HEM and $1.0 \mathrm{mN} / \mathrm{m}$ for NHEA-14. The surface morphologies of the domains are entirely different. The domains of 2-HEM are circular having internal texture, while those of NHEA-14 are dendritic having directional characteristics. The presence of interfacial hydrogen bonding in the latter amphiphile should be responsible for the formation of such characteristic domains in the case of latter amphiphile.
\end{abstract}

Key words: Langmuir monolayers, Phase transition, Brewster angle microscopy, 2-hydroxyethyl myristate, $n$-tetradecanoyl Nethanolamide.

\section{Introduction}

In the generally accepted membrane model, proteins are embedded in a lipid bilayer, which consists of two weakly coupled monolayers. Since Langmuir monolayers are easier to investigate and provide quantitative information, these monolayers of lipids particularly phospholipids have traditionally been studied as models of biological membranes (Möhwald, 1990). Research on phase behavior in spread or Langmuir monolayers of insoluble amphiphiles has been an interesting exercise since it provides new insights into the morphological features of domains formed during first-order phase transition from a liquid expanded (LE) phase to a liquid condensed (LC) phase in the monolayers, which are not common in three-dimensional systems. In the LC state, the polar group of the monolayer molecules is incorporated into the aqueous phase while the hydrocarbon part of the molecule is kept away from the water surface. Both the interactions between neighboring molecules and the interactions between molecules and the subphase are important for the molecular arrangement and the mesoscopic phase behavior, which can be studied by film balance measurement, and Brewster angle microscopy (BAM) (Hénon and Meunier, 1991).

\footnotetext{
* Corresponding author: E-mail: mdmhossain@yahoo.com.
}

Application of BAM permits us to visualize a large variety of shapes and textures of the domains in the spread monolayers (Rivière et. al., 1994; Hossain et. al., 2000; He and Li 2003; Overbeck et. al., 1994). Both the thermodynamic properties of the monolayers, shapes and textures of the LC domains are reported to be affected by a number of variables such as temperature (Hossain and Kato 2000; Islam and Kato 2003), chain length (Islam and Kato, 2006), nature of the head groups (Johann et. al., 2001), pH of the subphase (Fainerman et. al., 2000; Johann et. al., 2001), presence of metal ions (Ren et. al., 2001) etc. Among these variables, systematic study on the effect of head groups is of least concern. In most of the cases, shapes of the domains are circular at lower temperatures and fingering or fractal at higher temperatures (Hossain et. al., 2000; He and Li 2003; Overbeck et. al., 1994; Hossain and Kato 2000; Islam and Kato 2003). In some cases, absolutely opposite behavior of domain shapes have been reported recently (Hossain et.al. 2006; Islam and Kato, 2005). Although, these are related with temperature dependency, whether the system adopts normal or opposite behavior is somehow governed by the nature and size of the head groups. Even, the effect of $\mathrm{pH}$ or the effect 
of metal ions on the monolayer properties is related with the head group interactions which are altered by changing $\mathrm{pH}$ or by adding the metal ions (Fainerman et. al., 2000; Johann et. al., 2001 and Ren et. al., 2001). Thus, it is very important to study systematically the effect of head groups on the properties of the monolayers. In this paper, we carry out experiment by changing the head groups and taking all other variables such as temperature, chain length, $\mathrm{pH}$ etc constant. For this purpose, we choose two amphiphiles, 2-hydroxyethyl myristate (2-HEM) and n-tetradecanoyl N-ethanolamide (NHEA-14), having 13-carbon as tail (Figure 1). The only difference

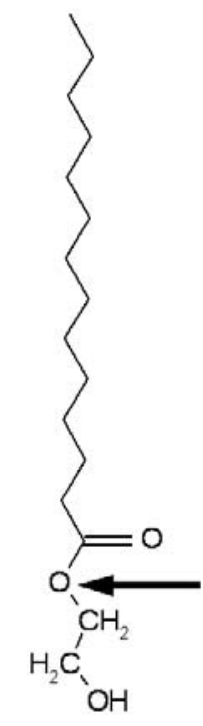

2-HEM

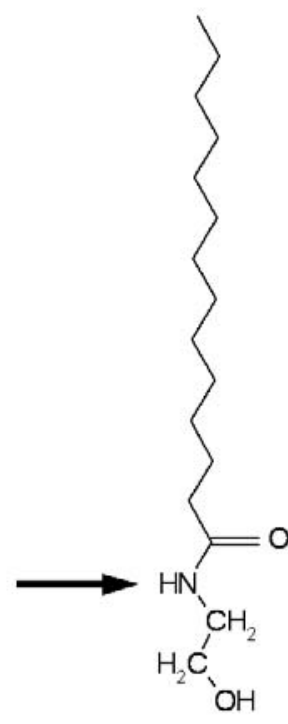

NHEA-14
Fig. 1: Structures of the amphiphiles. Arrows indicate the only sites of the differences between the two amphiphiles.

in the head group is that 2-HEM has an ester linkage and NHEA-14 has an amide linkage in their head groups. The aim of this paper is to show that only a small change in the head group is sufficient to cause a drastic change in the thermodynamic properties as well as morphological features of the LC domains formed in the monolayers.

\section{Materials and Methods}

Both the amphiphiles, 2-HEM and NHEA-14 were synthesized and purified in our laboratory (Japan). The purities of the samples were checked by $400 \mathrm{MHz}{ }^{1} \mathrm{H}$ NMR (Varian Unity INOVA) and were much higher than $99.5 \%$ because there was no small impurity peak detectable in comparison with the satellite peaks of ${ }^{13} \mathrm{C}$. Ultrapure water of resistivity $18 \mathrm{M} \Omega$-cm (Elgastat, UHQ-PS) was used throughout the present study.

All the experiments were performed in a home-built shallow-type Langmuir trough above which a BAM was mounted. The surface pressure was measured by the Wilhelmy method using a small rectangular glass plate. This plate was cleaned by immersion into $1 \% \mathrm{HF}$ acid followed by washing with ultra-pure water prior to each experiment. The temperature of the trough was controlled by using a large amount of integrated Peltier elements attached to the base plate of the trough, and was detected by a platinum wire temperature sensor. The BAM is composed of a $20 \mathrm{~mW}$ He-Ne laser, a Glan-Thompson polarizer, an analyzer, a zooming microscope, a CCD camera of high sensitivity and a video recording system. The images recorded were distorted due to the oblique glancing of the microscope, and were corrected by an image-processing soft-ware.

The $\pi$-A isotherms of the Langmuir monolayers were measured by the usual solvent spreading technique. Spectral grade hexane (Dojin Chemicals) was used as a solvent of the spreading solution to assure fast evaporation. After spreading the solution of the amphiphile, about $20 \mathrm{~min}$ is allowed to evaporate the solvent completely. The isotherms were recorded with a constant strain rate mode of compression (30 $\% \mathrm{~min}^{-1}$ ) controlled by a program with a computer.

\section{Results and Discussion}

Figure 2 represents the $\pi$-A isotherm of 2 -HEM at $10^{\circ} \mathrm{C}$. With compression, initially the surface pressure remains almost constant at about zero value. With a decrease in the area, the pressure rises continuously. At an area of 0.52 $\mathrm{nm}^{2} /$ molecule and a pressure of $6.0 \mathrm{mN} / \mathrm{m}$, the $\pi$-A isotherm shows a cusp point followed by a plateau. These characteristics are indicative of a first-order phase transition (Hossain et al., 2006; Islam and Kato, 2005). With a further decrease in the area, the surface pressure rises rapidly at an area about $0.25 \mathrm{~nm}^{2} /$ molecule. Since the phase transition appears between 0.52 and $0.25 \mathrm{~nm}^{2} /$ molecule, this transition is classified as liquid expanded (LE)-LC phase transition (Hossain et.al. 2006; Petty, 1996). Figure 3 represents the $\pi$-A isotherm of NHEA- 14 at $10{ }^{\circ} \mathrm{C}$. Similar to 2-HEM, the $\pi$-A isotherm of NHEA- 14 at $10^{\circ} \mathrm{C}$ show a first-order phase transition at a surface area of $0.52 \mathrm{~nm}^{2} /$ molecule and a surface 


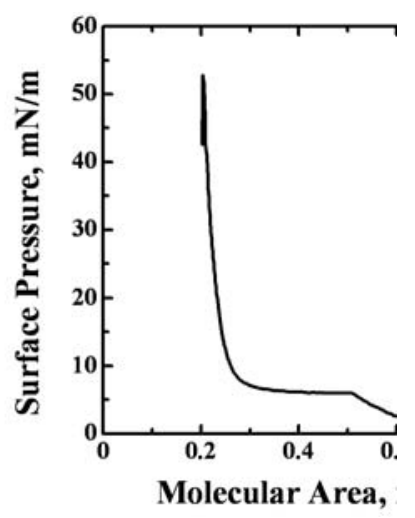

Fig. 2: $\pi \mathrm{A}$ isotherm of the Langmuir monolayer of 2HEM at $10{ }^{\circ} \mathrm{C}$.

pressure of $1.0 \mathrm{mN} / \mathrm{m}$. This phase transition is also classified as first-order LE-LC phase transition. At the same temperature, the surface pressure necessary for LE-LC phase transition is higher for 2-HEM than that of NHEA-14. This indicates that the attractive force among the head groups is higher for NHEA-14 which allows easy condensation of the LE phase into LC phase. Since, the amphiphiles are different only by the head groups, one has an ester group and the other has an amide group, the stronger attractive force in NHEA14 is due to the stronger hydrogen bonding capacity of this amphiphile. The limiting areas, calculated by extrapolating the steepest region of the isotherms, for both the cases are about $0.22 \mathrm{~nm}^{2} /$ molecule, which is consistent with those of the other similar molecules (Hossain et. al. 2006; Petty, 1996).

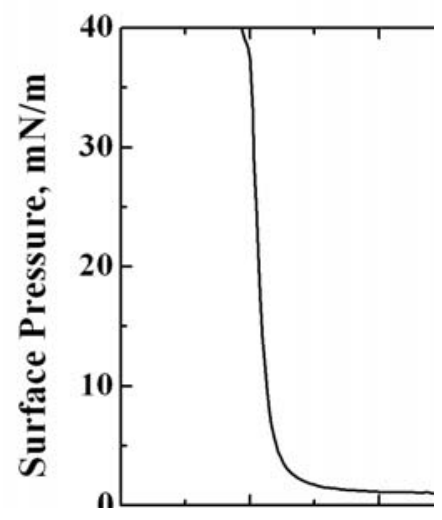

Fig. 3: $\quad \pi \mathrm{A}$ isotherm of the Langmuir monolayer of NHEA-14 at $10^{\circ} \mathrm{C}$
Figures 4 and 5 show the in situ BAM images observed during the compression of the monolayers. In both cases, the images show only one dark phase at the initial stage of compression. This phase is the LE phase. However, just after the cusp point in each of the $\pi$-A isotherms (both figures), bright phase appears in the dark background. This bright phase grows in size with compression and finally the surface is fully covered with this phase. The presence of two surface phases confirms the existence of first-order LE-LC phase transition, where the bright phase is the LC phase. When the surface is fully covered with this bright LC phase the area covered by the molecules cannot be reduced much rather the surface pressure rises very rapidly. The reason behind this is that at this point, the molecules are just touched each other and cannot come closer. Thus, the limiting molecular area, which is $0.22 \mathrm{~nm}^{2} /$ molecule is in fact the cross-sectional area of the head groups for both the amphiphiles. The most interesting features of LC domains formed during the phase transitions are that 2-HEM shows circular domains having internal texture, whereas NHEA-14 presents dendritic domain having directional characteristics. Although data are not shown here, the circular domains of 2-HEM remain unaltered, but dentritic domains of NHEA-14 show more sharpness of the arms with increasing temperature. Generally, the shapes of the LC domains are governed by the balance between the line tension, which is a measure of van der Waal's attractive force among the hydrophobic tails, and the dipole-dipole repulsion among the head groups. When the line tension dominates over the dipole-dipole repulsion, the LC domains have compact shape or circular shape. This happens in the case of 2-HEM. The presence of texture in the domains indicates that the molecules remain tilted. Since the contrast between the brightness of two adjacent segments is weak, the tilt angle of molecules should be small. This is also
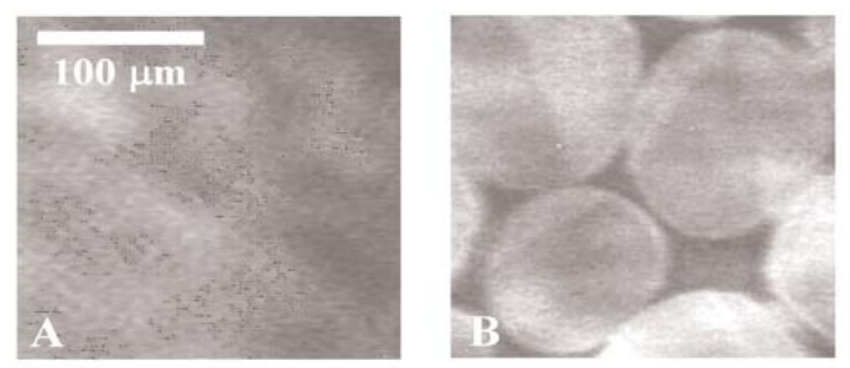

Fig. 4: BAM images of the Langmuir monolayers of 2-HEM observed in situ during compression at $10^{\circ} \mathrm{C}$ at different areas; (A) $0.6 \mathrm{~nm}^{2} / \mathrm{mole}$ cule (B) $0.29 \mathrm{~nm}^{2} /$ molecule 

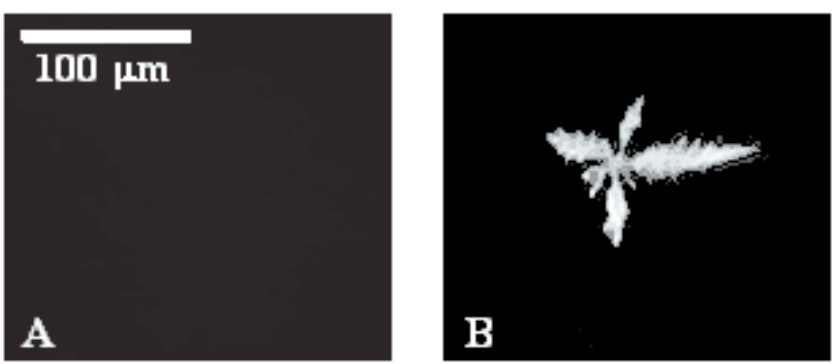

Fig. 5: BAM images of the Langmuir monolayers of NHEA-14 observed in situ during compression at $10{ }^{\circ} \mathrm{C}$ at different areas; (A) $0.6 \mathrm{~nm}^{2} / \mathrm{mole}-$ cule (B) $0.41 \mathrm{~nm}^{2} /$ molecule

expected from the view point of chain length containing only 13-carbon. On the other hand, the formation of dendritic shapes of the LC domains formed in NHEA-14 cannot be explained by the balance between the line tension and the dipole-dipole interactions. Vollhardt et al. (Vollhardt et al., 1996; Melzer et al.,1998) reported that amphiphiles having the capacity of formation of hydrogen bonding show dendritic domains with directional characteristics. Thus the presence of - $\mathrm{CONH}$ - group having the sites for hydrogen bonding close to the interface should be responsible for the formation of dendritic domains in the case of this amphiphile. Since, the hydrogen bonding occurs in a particular direction and is stronger than the line tension which is a result of van der Waal's interactions, this latter effect has very little to change the domain shapes. All of these explanations are shown by a model (Figure 6). This figure clearly predicts that in 2-HEM monolayer, the molecules come closer due to the van der Waal's attractive force among the hydrophobic tails. Whereas in NHEA-14 monolayers, the molecules come closer not only due to the van der Waal's attractive force but also by the strong interfacial hydrogen bonding by $-\mathrm{CONH}$ - group present in this amphiphile.

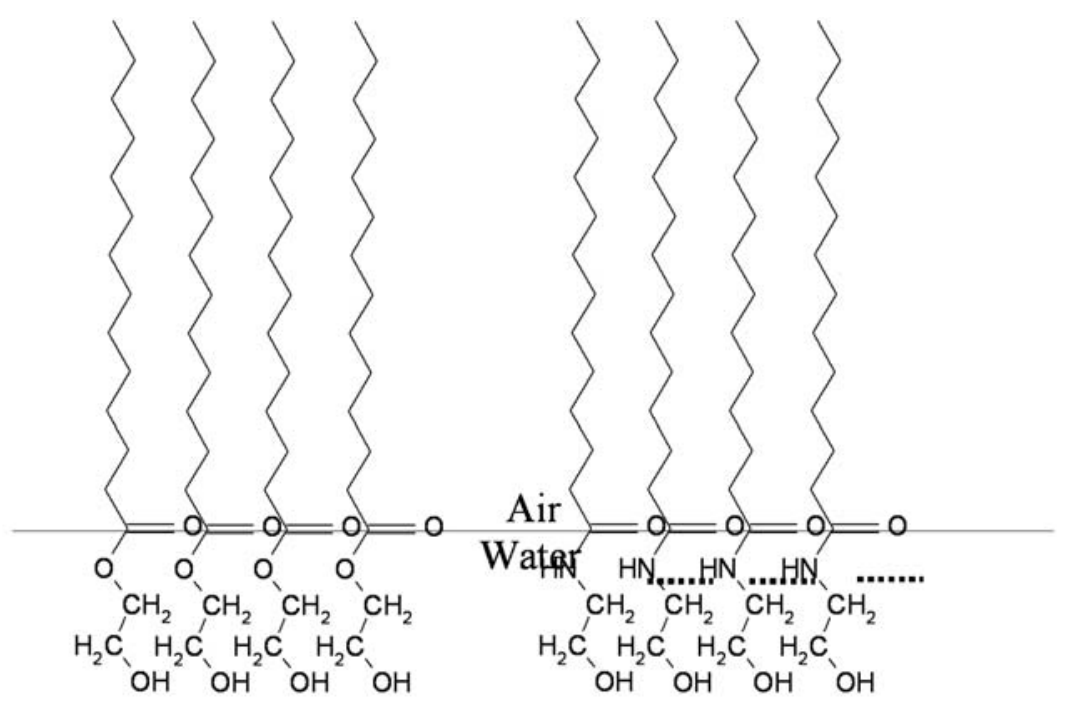

2-HEM

NHEA-14

Fig. 6: Models showing the difference of interactions between different head groups. The dashed line among the head groups indicates the interfacial hydrogen bonding 


\section{Conclusion}

In this paper, it has been ascertained that a small change in the head group causes a drastic change in both the thermodynamic properties and the morphological features of the LC domains formed in the Langmuir monolayers at the air-water interface. Both the amphiphiles show a first-order LE-LC phase transition at $10{ }^{\circ} \mathrm{C}$. However, the critical surface pressure necessary for the phase transition is $6.0 \mathrm{mN} / \mathrm{m}$ for 2 HEM whereas that for NHEA-14 is $1.0 \mathrm{mN} / \mathrm{m}$. The domains of 2-HEM are circular having internal texture, while those of NHEA-14 are dendritic having directional characteristics. The formation of such characteristic domains should be due to the presence of interfacial hydrogen bonding in the latter amphiphile.

\section{Acknowledgement}

The financial support from the venture business laboratory (VBL) of Utsunomiya University is gratefully acknowledged.

\section{References}

Fainerman V. B., Vollhardt D. and Johann R. (2000). Arachidic acid monolayers at high $\mathrm{pH}$ of the aqueous subphase: Studies of counter ion bonding. Langmuir, 16: 7731-7736.

He Q. and Li J. (2003). Dynamic and morphological investigation of phospholipid monolayer hydrolysis by phospholipase C. Biochem. Biophys. Res. Com, 300: 541545.

Hénon S. and Meunier J. (1991). Microscope at the Brewster angle: Diercet observation of first-order phase transitions in monolayers. Rev. Sci. Instrum., 62: 936-939.

Hossain M. M., Yoshida M., Iimura K., Suzuki N. and Kato T. (2000). Phase Transition in Adsorbed Monolayers of 2-Hydroxyethyl Laurate at the Air-Water Interface. Colloid Surf. A 171: 105-113.

Hossain M. M. and Kato T. (2000). Line tension Induced Instability of Condensed Domains Formed in Adsorbed Monolayers at the Air-Water Interface. Langmuir 16:
$10175-10183$.

Hossain M. M., Iimura K. and Kato T. (2006). Effect of Temperature on the Surface Phase Behavior of nHexadecyl Dihydrogen Phosphate in Adsorption Layers at the Air-Water Interface. J. Colloid Interface Sci. 298: 348-355.

Hossain M. M., Iimura K. and Kato T. (2006). Surface Phase Behavior of Di- $n$-tetradecyl Hydrogen Phosphate in Langmuir Monolayers at the Air-Water Interface. $J$. Colloid Interface Sci. 302: 272-277.

Islam M. N. and Kato T. (2003). Temperature Dependence of the Surface Phase Behavior and Micelle Formation of Some Nonionic Surfactants. J. Phys. Chem. B, 107: 965-971.

Islam M. N. and Kato T. (2005). Influence of Temperature and Headgroup Size on Condensed-Phase Patterns in Langmuir Monolayers of Some Oxyethylenated Nonionic Surfactants Langmuir 21: 2419-2424.

Islam M. N. and Kato T. (2006) Influence of temperature and alkyl chain length on phase behavior in Langmuir monolayers of some oxyethylenated nonionic surfactants. J. Colloid Interface Sci. 294: 288-294.

Johann R., Brezesinski G., Vollhardt D. and Möhwald H. (2001). The effect of headgroup interactions on structure and morphology of arachidic acid monolayers. $J$. Phys. Chem. B, 105: 2957-2965.

Johann, R., Vollhardt D. and Möhwald H. (2001). Shifting of fatty acid monolayer phases due to ionization of the head groups. Langmuir, 17: 4569-4580.

Melzer V., Vollhardt D., Brezesinski G. and Möhwald H. (1998). Similarities in the phase properties of Gibbs and Langmuir monolayers. J. Phys. Chem. B, 102: 591-597.

Möhwald H. (1990). Phospholipid and Phospholipid-Protein Monolayers at the Air/Water Interface Phospholipid and Phospholipid-Protein Monolayers at the Air/Water Interface. Annu. Rev. Phys. Chem. 41: 441-476. 
Overbeck G. A., Hönig D., Wolthaus L., Gnade M. and Möbius D. (1994). Observation of bond orientational order in floating and transferred monolayers with Brewster angle microscopy. Thin Solid Films, 242: 2632.

Petty M. C. (1996). Langmuir Blodgett Films, first ed., Cambridge Univ. Press, New York.

Ren Y., Hossain M. M., Iimura K. and Kato T. (2001). $\mathrm{CH}_{3}\left(\mathrm{CH}_{2}\right)_{n} \mathrm{COOH} / \mathrm{Cd}^{2+}$ System on the Aqueous Cadmium Acetate Solution Investigated in situ by Polarization Modulation Infrared Spectroscopy. $J$. Phys. Chem. B, 105: 7723-7729.
Rivière S., Hénon S. and Meunier J. (1994). Fluctuation of a defect line of molecular orientation in a monolayer. Phys. Rev. E, 49: 1375-1382.

Vollhardt D., Emrich G., Gutberlet T. and Fuhrhop J.-H. (1996). Chiral discrimination and pattern formation in $\mathrm{N}$-dodecylmannonamide monolayers at the air-water interface. Langmuir, 12: 5659-5663.

Received : January 03, 2010;

Accepted : March 16, 2010 